\title{
BMJ Open Internet-based cognitive-behavioural writing therapy for reducing post-traumatic stress after intensive care for sepsis in patients and their spouses (REPAIR): study protocol for a randomised-controlled trial
}

\author{
Romina Gawlytta, ${ }^{1,2}$ Helen Niemeyer, ${ }^{3}$ Maria Böttche, ${ }^{3,4}$ André Scherag, ${ }^{2}$ \\ Christine Knaevelsrud, ${ }^{3}$ Jenny Rosendahl ${ }^{1,2}$
}

To cite: Gawlytta R,

Niemeyer $\mathrm{H}$, Böttche $\mathrm{M}$, et al. Internet-based cognitivebehavioural writing therapy for reducing post-traumatic stress after intensive care for sepsis in patients and their spouses (REPAIR): study protocol for a randomisedcontrolled trial. BMJ Open 2017:7:e014363.

doi:10.1136/bmjopen-2016014363

- Prepublication history for this paper is available online. To view these files please visit the journal online (http://dx.doi.org/10.1136/ bmjopen-2016-014363).

CK and JR contributed equally.

Received 20 September 2016 Revised 22 December 2016 Accepted 25 January 2017

\section{CrossMark}

For numbered affiliations see end of article.

Correspondence to Dr Jenny Rosendahl; jenny.rosendahl@ med.uni-jena.de

\section{ABSTRACT}

Introduction: As a consequence of sepsis and intensive care, considerable proportions of patients but also of their spouses develop a post-traumatic stress disorder (PTSD). However, only a very small number receive psychotherapeutic treatment. Internet-based cognitive-behavioural writing therapy (IB-CBWT) has proven to be an effective treatment option for PTSD. It seems to fit the specific needs of this cohort and to overcome treatment barriers. Aim of the REPAIR trial is to examine the efficacy, safety and applicability of IB-CBWT for PTSD in patients and their spouses after intensive care for sepsis.

Methods and analysis: Participants will be assigned randomly either to a treatment or a wait-list (WL) control group. The treatment group receives IB-CBWT for PTSD, actively involving the partners of the participants. IB-CBWT will be guided by a therapist and comprises two written assignments per week over a 5 week period. After completing the assignments, the participants obtain individual responses from the therapist. Participants of the WL control group will receive treatment after a waiting period of 5 weeks. The primary outcome is PTSD symptom severity in self-rated PTSD Checklist for Diagnostic and Statistical Manual Fifth Edition at the end of treatment and waiting time, respectively. Secondary outcomes are remission of PTSD, depression, anxiety, and somatisation measured by the Brief Symptom Inventory-18, marital satisfaction measured by the Relationship Assessment Scale, health-related quality of life measured by the EQ5D-5L, and the feasibility of IB-CBWT for this cohort (ie, dropout rate). Statistical analysis will be performed according to the intent-to-treat principle.

Ethics and dissemination: The study is conducted according to the principles of Good Clinical Practice and has been approved by the ethics committee of the Friedrich-Schiller University Jena, Germany. Results will be disseminated at scientific conferences, published in peer-reviewed journals, and provided to consumers of healthcare.
Strengths and limitations of this study

This randomised-controlled trial will provide new evidence concerning the treatment of posttraumatic stress disorder (PTSD) after intensive care for sepsis in patients and their spouses.

- For the first time also the spouses of patients with PTSD will be involved in their partners' internet-based cognitive-behavioural writing therapy.

- Intervention effects will be compared against a wait-list control group.

- It is not possible to ensure a complete blinding of patients and therapists.

Trial registration number: Pre-results, DRKS00010676.

\section{INTRODUCTION}

Psychopathological reactions, that is, acute stress disorder (International Classification of Diseases 10th Revision: F43.0) and posttraumatic stress disorder (PTSD; F43.1), are common consequences of life-threatening events such as sepsis and negatively affect patients' long-term functioning and quality of life ${ }^{1-4}$ Critical illness can also be a traumatic and stressful experience for family members as a result of uncertainty and the fear of the patient's physical disability or death. Diagnostic and Statistical Manual Fifth Edition (DSM-5) $)^{5}$ explicitly defined the diagnostic criteria of a traumatic event as an exposure to actual death or serious injury experienced in person or which has occurred to a close family member. Family 
members, particularly spouses, who care for the critically ill patient during the time of intensive care, are therefore a vulnerable cohort. ${ }^{6-8}$ In a recent study, up to $69 \%$ of the patients, who had survived sepsis and $62 \%$ of the spouses of sepsis survivors suffered from clinically relevant PTSD symptoms. ${ }^{9}$ It has been further shown that both physical and mental health of patients and their spouses are interrelated. More specifically, results of a dyadic analysis indicated that the mental quality of life of a person (patient or spouse) is negatively impacted by post-traumatic stress symptoms of the respective partner. Furthermore, it has been shown that PTSD symptoms of the patient who survived sepsis are a significant predictor of PTSD symptoms of the respective spouse. ${ }^{10}$ Based on these results, it has been concluded that couples react as a dyadic system with interdependent emotional responses to critical illness. Thus, the inclusion of spouses in the treatment of mental longterm consequences of critical illness appears to be inevitable. ${ }^{9}$ However, patients suffering from PTSD after critical illness are often untreated or undertreated hereof. Accordingly, Mehlhorn et $a l^{11}$ suggest in their review of interventions for the postintensive care syndrome, that "postintensive care patients may benefit from interventions like trauma-focused cognitive-behavioural therapy [...] but often they do not have access to those interventions". (p. 1268)

With regard to the treatment of PTSD, several evidence-based interventions exist. There is striking evidence for the efficacy of trauma-focused cognitivebehavioural therapy with large effect sizes (standardised mean difference $=1.62 ; 95 \%$ CI $(1.21$ to 2.03$)$ in a meta-analysis of 28 studies) compared against wait-list (WL) control. ${ }^{12}$ Nevertheless, only a minority of individuals suffering from PTSD seeks psychological treatment due to different barriers (eg, fear of stigmatisation, embarrassment, lack of availability of specialised therapists). In recent years, internet-based interventions based on CBT techniques have overcome these face-to-face treatment barriers by treating mobility-impaired patients, being independent in space and time as well as easily accessible and due to visual anonymity being lowthreshold. ${ }^{13-15}$ The internet-based approach is usually based on a manualised, therapist-assisted treatment which is operationalised via written assignments. In general, treatment as well as the diagnostic screenings (before and after the treatment) are conducted without any face-to-face contact in a secure web portal. ${ }^{13} 16 \quad 17$ Meta-analytic evidence has proven the efficacy of internet-based cognitive-behavioural writing therapy (IB-CBWT) to be large (Hedges' $\mathrm{g}=0.95 ; 95 \%$ CI $(0.46$ to 1.43); 8 studies) in PTSD symptom reduction compared to WL control. ${ }^{15}$

Up to now, IB-CBWT has not been considered as a treatment approach for PTSD after critical illness and intensive care. Moreover, therapeutic approaches for PTSD that include spouses in addition to the patients are very scarce.
Objectives

Primary aims of the REPAIR trial are to investigate the efficacy, safety and applicability of IB-CBWT for posttraumatic stress after intensive care for sepsis in patients and their spouses compared to a WL control group and to assess maintenance of possible treatment gains at 3,6 and 12 months post-treatment. Second, the study aims at examining dyadic concordance in treatment effects, that is, indirect effects of the treatment in the respective spouse of the participant of the treatment. Third, the influence of dyadic coping on the treatment effects will be explored.

\section{METHODS AND ANALYSIS}

Study design and setting

REPAIR is a randomised-controlled, parallel group, superiority trial. The current study will be conducted at the Jena University Hospital, recruiting participants from German speaking countries (eg, Germany, Austria and Switzerland) at least 1 month after discharge from the intensive care unit (ICU). Participants will be contacted via telephone for initial screening and via internet for delivering the treatment and conducting assessments.

\section{Eligibility criteria}

We will include adult (18+ years) patients after intensive care ( $>5$ days) for sepsis ${ }^{18}$ and their spouses (married or cohabited) who are fluent in written German. A patient-spouse dyad will be included if at least one of them (patient or spouse or both) scores above the PCL-5 cut-off (score $\geq 33)^{19}$ for a presumptive PTSD diagnosis. PTSD should be based on a trauma, which is associated with the critical illness and/or ICU stay. Patients will be excluded, if they do not have a spouse. According to the German clinical guideline on the treatment of PTSD ${ }^{20}$ acute psychosis and suicidal ideation will be criteria for exclusion. Furthermore, the use of neuroleptics, or an ongoing psychotherapeutic treatment elsewhere will be reasons for exclusion.

\section{Procedures}

Recruitment

Since the treatment is delivered internet-based, German speaking patients/spouses could participate from all over the world. For recruitment, we follow a multipartite strategy. First, all persons, that is, patients or spouses, who request free of charge advice from the German Sepsis Aid's National Helpline (http://www.sepsis-hilfe. org) or had requested advice in the past 2 years (altogether about 600 requests), will be contacted and informed about the study. Second, patients of the Mid-German Sepsis Cohort (MSC; trial registration: German Clinical Trials Register, no. DRKS00010050) who are positively screened for PTSD at one of the MSC study assessments will be informed about the study. The MSC aims at following-up about 1000 patients after 
sepsis per year, of whom we expect about $20 \%$ to have PCL scores $\geq 33$ points at least at one follow-up assessment. Third, participants will be recruited via advertisements in health journals and distribution of information brochures in hospitals and rehabilitation centres. In a first telephone contact, participants will be screened for eligibility by using the Life Event Checklist for DSM-5 $\left(\right.$ LEC 5) ${ }^{21}$ and the PTSD checklist for DSM-5 (PCL-5). ${ }^{19}$ Written informed consent will be obtained by the patients and their spouses (see figure 1). One signed version of the informed consent will be sent back to the study centre. After that, an appointment for a second telephone interview will be terminated. In this second telephone contact, patients and their spouses will complete the Clinician-Administered PTSD Scale for DSM-5 $(\text { CAPS-5 })^{22}$ and the Structured Clinical Interview for DSM-IV (SCID-I) ${ }^{23}$ conducted by a trained psychologist. Medical data will be assessed (eg, length of intensive care and (if) length of mechanical ventilation, time since ICU discharge).

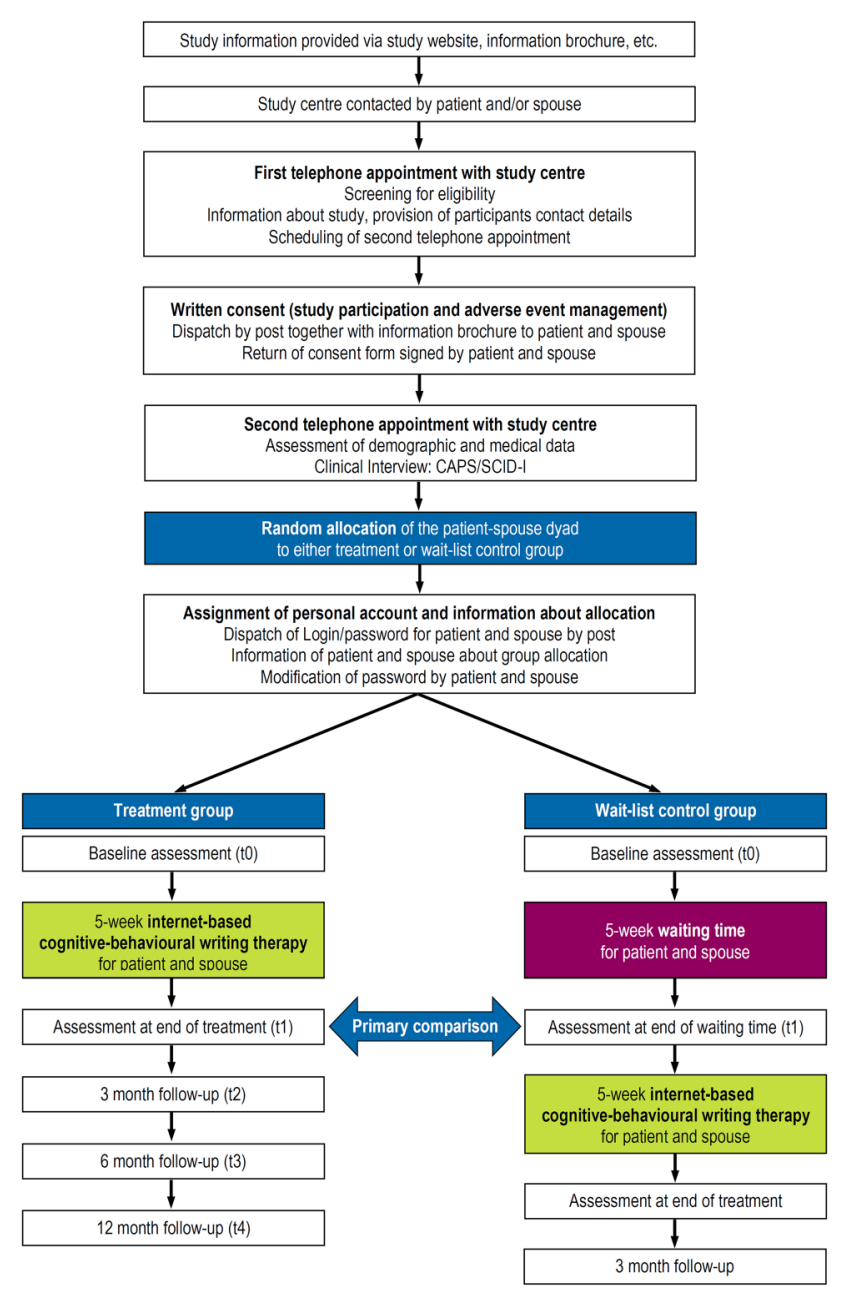

Figure 1 Study flow chart. CAPS/SCID-I, ClinicianAdministered PTSD Scale for DSM-5/Structured Clinical Interview for DSM-IV DSM, Diagnostic and Statistical Manual; PTSD, post-traumatic stress disorder.
Randomisation

All eligible patient-spouse dyads consenting to participation will be randomly assigned to either IB-CBWT or to a WL control group (allocation ratio 1:1) with the patient-spouse dyad being the unit of randomisation. Randomisation will be conducted using a central internet-based registration system provided by the Center for Clinical Studies of the Jena University Hospital. This system automatically randomises patients and generates a message noting the assigned treatment. The underlying randomisation list will be developed by an independent biometrician using a computer-based algorithm. Allocation will be concealed and stratified by the occurrence of PTSD symptoms within the dyads of sepsis survivor and the spouse: strata 1 -both, sepsis survivor and spouse with PTSD; strata 2-sepsis survivor with PTSD/spouse without, and strata 3-spouse with $\mathrm{PTSD} /$ sepsis survivor without.

\section{Baseline assessment (t0)}

Before the start of the treatment participants, that is, patients and their spouses, will be asked to complete the following questionnaires: PTSD checklist for DSM-5 (PCL-5) ${ }^{19}$ Brief Symptom Inventory (BSI),${ }^{24}$ Resilience Scale (RS13), ${ }^{25}$ Proactive Coping Inventory (PCI), ${ }^{26}$ Multidimensional Fatigue Inventory (MFI), ${ }^{27}$ EQ-5D-5L health questionnaire, ${ }^{28}$ Index for Measuring Limitations of Social Participation (IMET), ${ }^{29}$ Dyadic Coping Inventory (DCI), ${ }^{30}$ Relationship Assessment Scale $(\text { RAS })^{31}$ and Internet Literacy Questionnaire (ILQ; subscale technical expertise).$^{32}$ Additionally, Posttraumatic Cognitions Inventory (PTCI) ${ }^{33}$ will be assessed only in participants with PCL scores $\geq 33$ points (table 1 ).

\section{Intervention phase}

\section{Internet-based writing therapy}

Patients and/or spouses with PCL scores $\geq 33$ points, who are allocated to the treatment condition, will participate in an IB-CBWT. They will be asked to complete two 50 min writing assignments per week over a 5 -week period (10 essays in total). The therapy consists of three treatment modules (table 2): (1) resource-oriented biographical reconstruction (three essays), (2) in sensu trauma exposure sessions (four essays) and (3) cognitive reconstruction (three essays).

Integrated in the third module, the respective partner of the treated participant diagnosed with PTSD receives instructions to write a supportive letter to him/her. Here, the respective partner should announce acknowledgement for the participant as well as his/her strengths and the shared future. Partners without clinically relevant PTSD symptoms will also receive access to an individual web portal where they complete the assessments and write the supportive letter. They further receive psychoeducational information about mental health problems after traumatic events (ie, explanation of PTSD symptoms and treatment options). 
Table 1 Schedule of the assessments

\begin{tabular}{|c|c|c|c|c|c|c|c|c|}
\hline \multirow[b]{3}{*}{ Timepoint } & \multicolumn{8}{|c|}{ Study period } \\
\hline & \multirow{2}{*}{$\begin{array}{l}\text { Enrolment } \\
\text { tx }\end{array}$} & \multirow{2}{*}{$\begin{array}{l}\text { Allocation } \\
\text { to }\end{array}$} & \multicolumn{3}{|c|}{ Intervention } & \multicolumn{3}{|c|}{ Follow-up } \\
\hline & & & $\overline{\text { S3 }}$ & S7 & t1 & t2 & t3 & t4 \\
\hline \multicolumn{9}{|l|}{ Enrolment } \\
\hline Informed consent & $x$ & & & & & & & \\
\hline Eligibility screen & $x$ & & & & & & & \\
\hline Allocation & & $x$ & & & & & & \\
\hline \multicolumn{9}{|l|}{ Interventions } \\
\hline \multicolumn{9}{|l|}{ IB-CBWT } \\
\hline \multicolumn{9}{|l|}{ Wait-list control group } \\
\hline \multicolumn{9}{|l|}{ Assessments } \\
\hline $\begin{array}{l}\text { Demographic and medical } \\
\text { information }\end{array}$ & & $x$ & & & & & & \\
\hline ILQ & & $x$ & & & & & & \\
\hline CAPS-5 & & $x$ & & & $x$ & & & \\
\hline SCID-I & & $x$ & & & & & & \\
\hline PCL-5 & $x$ & $x$ & $x$ & $x$ & $x$ & $x$ & $x$ & $\mathrm{x}$ \\
\hline LEC-5 & & $x$ & & & & & & \\
\hline $\mathrm{BSI}$ & & $x$ & $x$ & $x$ & $x$ & $x$ & $x$ & $x$ \\
\hline RAS & & $x$ & $x$ & $x$ & $x$ & $x$ & $x$ & $x$ \\
\hline IMET & & $x$ & & & $x$ & $x$ & $x$ & $x$ \\
\hline RS-13 & & $x$ & & & $x$ & $x$ & $x$ & $x$ \\
\hline EQ-5D-5L & & $x$ & & & $x$ & $x$ & $x$ & $x$ \\
\hline MFI & & $x$ & & & $x$ & $x$ & $x$ & $x$ \\
\hline $\mathrm{DCl}$ & & $x$ & & & $x$ & $x$ & $x$ & $x$ \\
\hline PTCl & & $x$ & & & $x$ & $x$ & $x$ & $x$ \\
\hline $\mathrm{PCl}$ & & $x$ & & & $x$ & $x$ & $x$ & $x$ \\
\hline SEWIP* & & & $x$ & $x$ & $x$ & & & \\
\hline Adverse events & & & $x$ & $x$ & $x$ & & & \\
\hline \multicolumn{9}{|c|}{$\begin{array}{l}\text { *SEWIP is only applied to patients with PCL scores } \geq 33 \text {. } \\
\text { BSI, Brief Symptom Inventory; CAPS-5, Clinician-Administered PTSD Scale for DSM-5; DCI, Dyadic Coping Inventory; EQ-5D-5L, health } \\
\text { questionnaire of the EuroQol group; IB-CBWT, Internet-based cognitive-behavioural writing therapy; ILQ, Internet Literacy Questionnaire; } \\
\text { IMET, Index for Measuring Limitations of Social Participation; LEC-5, Life Event Checklist for DSM-5; MFI, Multidimensional Fatigue Inventory; } \\
\text { PCI, Proactive Coping Inventory; PCL-5, Post-traumatic stress disorder checklist; PTCI, Post-traumatic Cognitions Inventory; RAS, } \\
\text { Relationship Assessment Scale; RS13, Resilience Scale; S3, after treatment session 3; S7, after treatment session 7; SCID-I, Structured } \\
\text { Clinical Interview for DSM-IV; SEWIP, Multiperspective Assessment of General Change Mechanisms in Psychotherapy; t0, Baseline, before } \\
\text { start of treatment/waiting; t1, after end of treatment/waiting; t2, } 3 \text { months after end of treatment; t3, } 6 \text { months after end of treatment; t4, } \\
12 \text { months after end of treatment, (t2-t4 only for intervention group); tx, time of enrolment. }\end{array}$} \\
\hline
\end{tabular}

At the beginning of each writing assignment, participants propose individual timetables as to when they plan to write. After completion of each assignment, therapists provide individual feedback and further writing instructions within one workday. Important aspects of this feedback are acknowledgement of the participant's courage to disclose and describe their traumatic experiences, reinforcement of the participant's work on the essays, positive feedback and motivation and frequent summaries and encouragement of participants to voice their questions and doubts. Study participants will complete writing assignments through a secure web portal, ensuring that all correspondence is confidential and encrypted. Communication between participants and their therapist will occur asynchronously.

Every participant (patient and spouse) will receive access to a private, secure user account within the web portal. During treatment, all communication will be conducted within this account. Additionally, the therapist accounts are located in the web portal being secure and only accessible for the therapists. A database located at the server of the Jena University Hospital is connected with the web-portal, saving data using anonymous codes meeting the highest security standards.

\section{Therapists}

Therapists will be licensed clinical psychologists with previous experience in IB-CBWT. They will receive specialised training in the administration of the treatment and will be supervised continuously throughout the trial. Participants will be consecutively assigned to the therapists. When both, patient and spouse, have clinically relevant PTSD symptoms, they will have different therapists.

\section{Measurement during the course of treatment}

During treatment, that is, after assignments 3, 7 and 10, the Multiperspective Assessment of General Change Mechanisms in Psychotherapy (SEWIP), ${ }^{34}$ measuring resource activation, problem actuation, mastery, clarification of meaning, emotional bond and agreement on 
Table 2 Framework of the 10 writing assignments delivered during IB-CBWT after sepsis for patients and their spouses

\begin{tabular}{|c|c|c|c|}
\hline Session number & Session goals & Suggested structure & Suggested tools \\
\hline $1-3$ & $\begin{array}{l}\text { Resource-oriented biographical } \\
\text { reconstruction. }\end{array}$ & $\begin{array}{l}\text { Explaining the reason of the } \\
\text { reconstruction. } \\
\text { Provide a list of life-events. } \\
\text { Provide a summary and give } \\
\text { individual feedback. }\end{array}$ & $\begin{array}{l}\text { Provide list of possible important } \\
\text { personal life events } \\
\text { "What problems did you have and } \\
\text { how do you solve it?" }\end{array}$ \\
\hline $4-7$ & $\begin{array}{l}\text { In sensu exposure. } \\
\text { Detailed description of the trauma } \\
\text { with all sensations. }\end{array}$ & $\begin{array}{l}\text { Explain the need of exposure. } \\
\text { Explain how to describe the } \\
\text { trauma in a written way. } \\
\text { Provide a summary and give } \\
\text { individual feedback. }\end{array}$ & $\begin{array}{l}\text { Provide a list of questions due to } \\
\text { the traumatic event and the } \\
\text { sensations. }\end{array}$ \\
\hline $\begin{array}{l}\text { Text of partner } \\
\text { (between } 7 \text { and } 8 \text { ) }\end{array}$ & $\begin{array}{l}\text { Supportive letter: acknowledgment } \\
\text { of traumatic event. } \\
\text { Strength of partner. } \\
\text { Joint future. }\end{array}$ & $\begin{array}{l}\text { Explaining reason of } \\
\text { participation. Explain the } \\
\text { session goals. }\end{array}$ & $\begin{array}{l}\text { Provide a list of questions due to } \\
\text { the goals of the letter. }\end{array}$ \\
\hline $8-10$ & $\begin{array}{l}\text { Cognitive reconstruction: writing a } \\
\text { letter to an imaginary friend. } \\
\text { Writing a letter to oneself. }\end{array}$ & $\begin{array}{l}\text { Explaining reason of } \\
\text { reconstruction. } \\
\text { Explain session goals. } \\
\text { Provide a summary and give } \\
\text { individual feedback. }\end{array}$ & $\begin{array}{l}\text { Provide a list of questions due to } \\
\text { the goals of the letter. } \\
\text { eg, "Has something positive } \\
\text { resulted from the events?" }\end{array}$ \\
\hline
\end{tabular}

collaboration, will be applied to participants of the IB-CBWT group. Additionally, PCL-5, BSI and RAS will be administered during therapy (after assignments 3 and 7 ).

\section{Measurement at the end of treatment/waiting (t1)}

At the end of treatment or waiting time, respectively, the following measures will be applied to the participants: PCL-5, BSI, RS13, PCI, MFI, EQ-5D-5L, IMET, DCI and RAS. Again, PTCI will be assessed only in participants with PCL scores $\geq 33$ points (table 1 ). Additionally, participants will be interviewed by using the ClinicianAdministered PTSD Scale for DSM-5 (CAPS-5).

\section{Wait-list control group}

Treatment effects will be compared against a WL control group to allow for the provision of care (if delayed) to all trial participants. After 5 weeks of waiting (duration of treatment), participants allocated to the $\mathrm{WL}$ control group will receive IB-CBWT. During and after this delayed application of IB-CBWT, the same measures as in the treatment condition will be assessed. However, these participants will not receive a supportive letter from their spouses. This will allow for evaluating the effect ascribed to the supportive letter.

\section{Follow-up phase}

Participants assigned to the treatment group will be followed up 3, 6 and 12 months after treatment, respectively. Participants assigned to the $\mathrm{WL}$ control group will be followed up 3 months after treatment. Outcome measures will be assessed again (table 1).

\section{Discontinuation}

If a participant meets any of the following criteria, the study intervention will be discontinued: withdrawal of consent to receive the study intervention, emergence of an adverse event (suicidal ideation, severe symptom increase) or start of psychotherapy elsewhere. The participant will be invited to continue completing the planned assessments. If participants withdraw consent to study participation, they will not be contacted for assessments in the future. Participants have the right to initiate deletion of their study data. If a participant does not make use of this right, all data will be included in the analyses.

If either the spouse or patient drops out of the study for any reason, the other participant will be allowed to continue with the intervention and study participation.

\section{Outcome measures \\ Primary outcome}

Primary outcome is the change in PTSD symptom severity score from baseline to 5 weeks after randomisation ( 11 ; at the end of treatment/waiting time) measured via the PTSD Checklist (PCL-5) covering the four DSM-5 clusters. ${ }^{19}$

\section{Secondary outcomes}

Secondary outcomes will be remission at $\mathrm{t} 1$ and the percentage of participants leaving the study early (during treatment phase) due to any reason (until t1). Furthermore, anxiety, depression and somatisation (Brief Symptom Inventory-18 $8^{24}$ ), marital satisfaction (Relationship Assessment Scale ${ }^{31}$ ) and health-related quality of life (EQ-5D-5 $\mathrm{L}^{28}$ ) all measured as summary scores at $\mathrm{t} 1$ and at follow-up (t2-t4: 3, 6, and 12 months).

\section{Other measures}

Additionally, we will assess dyadic coping with stress in the patient-spouse dyads using the Dyadic Coping 
Inventory, ${ }^{30}$ coping with stress on an individual level using the Proaactive Coping Inventory, ${ }^{26}$ social participation using the Index for Measuring Limitations of Social Participation, ${ }^{29}$ resilience (defined as the capacity to withstand life stressors and to thrive and make meaning from challenges ${ }^{35}$ ) using the Resilience Scale-13, ${ }^{25}$ fatigue using the Multidimensional Fatigue Inventory ${ }^{27}$ and post-traumatic cognitions using the Posttraumatic Cognitions Inventory. ${ }^{33}$ All of these measures will be applied at baseline ( $\mathrm{t} 0)$, at the end of treatment/waiting time (t1) and at follow-up (t2-t4). During (S3, S7) and at the end of treatment ( $\mathrm{t} 1$ ), we will assess common therapeutic factors in patients with PCL scores $\geq 33$ using the Multiperspective Assessment of General Change Mechanisms in Psychotherapy. ${ }^{34}$

\section{Sample size estimation}

The sample size calculation is based on the parametric evaluation of a two-group comparison using Students' t-test, though a more complex statistical model will be used as the primary test. To detect large effect sizes as revealed by a meta-analysis, ${ }^{15}$ that is, effects of Cohen's $\mathrm{d}=0.95$, while requiring $\alpha=0.05$ (two-sided) while aiming at a comparison-wise power of $1-\beta=0.9$ (a higher power was chosen to address the problem that a more complex statistical analysis will be used), a sample size of $\mathrm{n}=2 \times 34=68$ patient-spouse dyads is necessary for the intent-to-treat (ITT) analysis. Dropout rates in IB-CBWT are encouragingly low; in a previous study with older adults $(65+$ years, comparable in age to the population of the proposed study), $89 \%$ of the participants completed every step of treatment. ${ }^{16}$ However, additional dropouts in a sample of sepsis survivors may be due to medical reasons, that is, health impairment or sudden death. Thus, we decided to increase the power by assuming a dropout rate of $30 \%$, so that altogether 98 dyads have to be randomised to either IB-CBWT or WL control group.

\section{Methods against bias}

Selection bias will be minimised by random and concealed central allocation of the patient-spouse dyads to treatment and control group using a centralised randomisation by the Center for Clinical Studies of the Jena University Hospital. However, performance bias might not be ruled out because blinding of patients/ spouses could not be realised due to intervention characteristics. Similarly, therapists cannot be fully blinded to group assignment since participants receiving treatment the first weeks of recruitment must have been automatically allocated to the treatment group. Treatments will be carefully manualised and predefined in terms of the content and number of sessions. To assure treatment fidelity, verbatim scripts of the correspondence between participants and therapists will be reviewed. Treatment fidelity checks will be performed based on a random selection of $30 \%$ of treatment sessions. Data will be analysed using an ITT approach. To ensure data quality, diagnoses will be made on the basis of a validated clinical interview conducted by a clinically experienced and trained psychologist. Questionnaires that will be used in the proposed study have been proven to be psychometrically sound instruments. To reduce the risk of sampling bias and to assure external validity, we will use a multipartite recruitment strategy and apply less restrictive eligibility criteria.

\section{Statistical analyses}

The primary end point of the efficacy assessment (PCL-5 change score at the end of the treatment, t1, ie, $\sim 6$ weeks after randomisation; relative to the randomisation t0) will be compared between both groups (ie, experimental group and WL control group). The null hypothesis $\mu_{\mathrm{EXP}}=\mu_{\mathrm{WL}}$, which implies that the PCL-5 change scores are identical in expectation, will be tested against the (two-sided) alternative hypothesis that there will be a difference between the groups $\left(\mu_{\mathrm{EXP}} \neq \mu_{\mathrm{WL}}\right)$. The confirmatory analysis will be performed in the ITT population. These hypotheses will be tested using a general linear model for the primary outcome and the group factor adjusted for PCL-5 at baseline (t0) with generalised estimating equations component to address the possible intradyad clustering. The null hypothesis will be rejected when the two-sided $\mathrm{p}$ value for the group variable is equal to or less than the two-sided significance level $\alpha=0.05$. The average mean difference in the PCL-5 change scores at t 1 is assumed to be clinically relevant when the mean PCL-5 score is more than 10 points lower for the experimental group than for the WL control group. ${ }^{19}$

We will address missing values by replacing all missing change scores with the worst change observed. Furthermore, we will explore the potential impact of dropouts (ie, missingness not completely at random) on the results in sensitivity analyses that will be outlined in the statistical analysis plan (SAP).

Additionally, there will be sensitivity analyses, for example, in the per-protocol (PP) population or stratified by patient and spouse. All additional analyses and the analyses of secondary end points will be carried out exploratively, that is, without adjustment for multiplicity. We will use adequate standard descriptive and inferential statistical techniques that are described in detail in the SAP. For the third explorative objectivedyadic interference in mental health-we will use a longitudinal Actor-Partner-Interdependence Model. To examine the impact of dyadic coping on treatment effects, we will extend the previously applied regression models.

\section{Data collection and management \\ Data collection}

Relevant data will be collected via telephone and using questionnaires delivered via the web-portal. Telephonically assessed data will be documented in writing and transferred to the study management software 'OpenClinica'. Data assessed by using standardised 
questionnaires within the web-portal, will be collected via a secure network (HTTPS) using input forms in the web browser. Data will be saved by using anonymised codes on a server of the Jena University Hospital ensuring highest safety standards.

\section{Data management}

Data management will be conducted by using the study management software 'OpenClinica' meeting common regulatory requirements (GCP, 21CFRPart11). To ensure a pseudonymised data analysis, every participant will receive a distinct ID. Data will be checked regularly for accuracy, implausible or missing data will be enquired in the study centre.

\section{Study monitoring}

The current study will be monitored by an independent data manager of the Centre for Clinical Studies of the Jena University Hospital including periodic inspections of the completeness and correctness of study documents and study data.

\section{Premature termination of the study}

Reasons for a premature termination of the study will be unjustifiable risks of continuation, new scientific findings during study duration or inadequate recruiting rate. Decision about discontinuation will be taken jointly by the principal investigators, the study biometrician and the Data Safety and Monitoring Board.

\section{Reporting of adverse events}

Assessment of safety will include recording any adverse effects during the treatment period by asking participants for experienced adverse events at the end of the treatment. In addition, during treatment participants are provided a telephone contact for emergency cases. In such a case, adverse events will be documented by the study team.

\section{Ethical considerations and dissemination Informed consent}

All eligible participants will be informed orally by a trained clinical psychologist about aims, content, procedure and length of the study; and about any potential risks and advantages in a true manner. After providing the opportunity to ask questions, written consent will be obtained by sending the informed consent document back to the study centre. Participants further receive a brochure with detailed information about the study. Participation is voluntary at any time. Participants will be informed about the voluntariness of study participation and the opportunity to interrupt or prematurely terminate study participation without giving reasons.

\section{Ethics review}

The study has been approved by the ethics committee of the Friedrich-Schiller University Jena, Germany (number 4777-04/16, 11 May 2016). The trial is registered in the
German Clinical Trials Register (DRKS); number DRKS00010676. Modifications in the study protocol will be communicated to the ethics committee as well as the DRKS.

\section{Access to data}

Principal investigators and the study statistician will have access to the final data set. To ensure confidentiality, data dispersed to project team members will be blinded of any identifying participant information.

\section{Dissemination}

Results of this study will be presented at scientific conferences and published in peer-reviewed journals. Furthermore, we will disseminate results and conclusions to consumers of healthcare. The study will be implemented and reported in line with the CONSORT statement. Authorship is granted to authors who make important contributions to the creation of the final publication.

\section{DISCUSSION}

This study aims to provide new evidence of treatment approaches particularly designed for patients after critical illness such as sepsis. The current study also involves the spouse of the affected patient since critical illness has consequences not only for the patient itself, but also for his/her spouse who shares concerns, sorrows and problems.

The limitation of this study is that the intervention effects will be compared against a WL control group which might overestimate the efficacy of the treatment to a certain degree. ${ }^{36}$ This will be taken into account in the interpretation of the results. Moreover, evidencebased treatment approaches of in post-ICU patients are rare. ${ }^{11}$ This argues against an active control condition. Alternatively, psychological treatment placebo faces the problem that the development of such a control condition in PTSD trials 'is very difficult, if not impossible'. ${ }^{12}$

Moreover, performance bias will possibly influence the effects since participants cannot be blinded because they are aware of their group allocation. Additionally, therapists will not be blinded to group assignment. However, manualisation of the treatment and treatment fidelity checks will counter the risk of bias.

Despite these limitations, this is the first randomised controlled trial to assess the efficacy, safety and applicability of an IB-CBWT after sepsis in patients and their spouses. Given the sparse number of existing treatment approaches for this group of patients IB-CBWT might be a valuable addition in the treatment of PTSD after sepsis. The results of this study will hopefully improve healthcare after sepsis for patients and their spouses. Given the efficacy, safety and applicability of this approach, the treatment could be easily transferred to other languages and thereby disseminated internationally. 
Author affiliations

${ }^{1}$ Institute of Psychosocial Medicine and Psychotherapy, Jena University Hospital, Jena, Germany

${ }^{2}$ Integrated Research and Treatment Center, Center for Sepsis Control and Care, Jena University Hospital, Jena, Germany

${ }^{3}$ Department of Clinical Psychological Intervention, Freie Universität Berlin, Berlin, Germany

${ }^{4}$ Berlin Center for Torture Victims, Zentrum ÜBERLEBEN, Berlin, Germany

Acknowledgements The authors would like to thank Ulrike Redlich and Monique Vogel from the German Sepsis Aid for their support in recruitment. Thanks are also due to Thomas Lehmann for providing the randomisation tool and Margit Leitner for her help with the design of the information material and the website. The authors very much appreciate the informatics support provided by Uwe Ziegler, Cornelia Baumgart, Sebastian Burchert and Florian Rissner. The authors would also like to thank Cornelia Platzer, Stephanie Platzer, Isabella Schiller and Cornelia Eichhorn for their support regarding the study management.

Contributors JR and CK conceived and designed the study, and drafted the grant proposal. RG and JR drafted the protocol of the study, and organise and supervise study implementation. HN, MB, AS and CK refined the study protocol and study implementation. $\mathrm{HN}, \mathrm{MB}$ and CK developed the treatment manual. AS provides methodological and statistical expertise. CK supervises the therapists. All authors critically reviewed and approved the final version of the manuscript.

Funding This study is funded by the German Federal Ministry of Education and Research, grant number $01 \mathrm{E} 01002$.

Competing interests None declared.

Provenance and peer review Not commissioned; externally peer reviewed.

Open Access This is an Open Access article distributed in accordance with the Creative Commons Attribution Non Commercial (CC BY-NC 4.0) license, which permits others to distribute, remix, adapt, build upon this work noncommercially, and license their derivative works on different terms, provided the original work is properly cited and the use is non-commercial. See: http:// creativecommons.org/licenses/by-nc/4.0/

\section{REFERENCES}

1. Davydow DS, Gifford JM, Desai SV, et al. Posttraumatic stress disorder in general intensive care unit survivors: a systematic review. Gen Hosp Psychiatry 2008;30:421-34

2. Needham DM, Davidson J, Cohen $\mathrm{H}$, et al. Improving long-term outcomes after discharge from intensive care unit: report from a stakeholders' conference. Crit Care Med 2012;40:502-9.

3. Parker AM, Sricharoenchai T, Raparla S, et al. Posttraumatic stress disorder in critical illness survivors: a metaanalysis. Crit Care Med 2015;43:1121-9.

4. Boer KR, van Ruler O, van Emmerik AA, et al. Factors associated with posttraumatic stress symptoms in a prospective cohort of patients after abdominal sepsis: a nomogram. Intensive Care Med 2008;34:664-74.

5. American Psychiatric Association. Diagnostic and Statistical Manual of Mental Disorders (DSM-5尺). 5th edn. Washington DC: American Psychiatric Association, 2013.

6. Hickman RL Jr, Douglas SL. Impact of chronic critical illness on the psychological outcomes of family members. AACN Adv Crit Care 2010;21:80-91.

7. Davidson JE, Jones C, Bienvenu OJ. Family response to critical illness: postintensive care syndrome-family. Crit Care Med 2012;40:618-24.

8. Petrinec AB, Daly BJ. Post-traumatic stress symptoms in post-ICU family members: review and methodological challenges. West $J$ Nurs Res 2016;38:57-78.

9. Rosendahl J, Brunkhorst FM, Jaenichen D, et al. Physical and mental health in patients and spouses after intensive care of severe sepsis: a dyadic perspective on long-term sequelae testing the Actor-Partner Interdependence Model. Crit Care Med 2013;41:69-75.

10. Wintermann GB, Weidner K, Strauss B, et al. Predictors of posttraumatic stress and quality of life in family members of chronically critically ill patients after intensive care. Ann Intensive Care 2016:6:69.

11. Mehlhorn J, Freytag A, Schmidt K, et al. Rehabilitation interventions for postintensive care syndrome: a systematic review. Crit Care Med 2014;42:1263-71.

12. Bisson Jl, Roberts NP, Andrew M, et al. Psychological therapies for chronic post-traumatic stress disorder (PTSD) in adults. Cochrane Database Syst Rev 2013;(12):CD003388.

13. Knaevelsrud C, Maercker A. Internet-based treatment for PTSD reduces distress and facilitates the development of a strong therapeutic alliance: a randomized controlled clinical trial. BMC Psychiatry 2007;7:13.

14. van Emmerik AA, Reijntjes A, Kamphuis $\mathrm{JH}$. Writing therapy for posttraumatic stress: a meta-analysis. Psychother Psychosom 2013;82:82-8.

15. Küster A, Niemeyer $\mathrm{H}$, Knaevelsrud $\mathrm{C}$. Internet-based interventions for posttraumatic stress: a meta-analysis of randomized controlled trials. Clin Psychol Rev 2016;43:1-16.

16. Knaevelsrud C, Böttche M, Pietrzak R, et al. Integrative testimonial therapy: an Internet-based, therapist-assisted therapy for German elderly survivors of the World War II with posttraumatic stress symptoms. J Nerv Ment Dis 2014;202:651-8.

17. Knaevelsrud C, Brand J, Lange A, et al. Web-based psychotherapy for posttraumatic stress disorder in war-traumatized Arab patients: randomized controlled trial. J Med Internet Res 2015;17:e71.

18. Singer M, Deutschman CS, Seymour CW, et al. The Third International Consensus Definitions for Sepsis and Septic Shock (Sepsis-3). JAMA 2016;315:801-10.

19. Weathers FW, Litz BT, Keane TM, et al. The PTSD Checklist for DSM-5 (PCL-5). National Center for PTSD, 2013. http://www.ptsd.va gov

20. Flatten G, Gast U, Hofmann A, et al. [S3-guideline for posttraumatic stress disorder ICD-10: F43.1]. Stuttgart: Schattauer, 2013.

21. Weathers FW, Blake DD, Schnurr PP, et al. The Life Events Checklist for DSM-5 (LEC-5). National Center for PTSD, 2013. http:// www.ptsd.va.gov

22. Weathers FW, Blake DD, Schnurr PP, et al. The Clinician-Administered PTSD Scale for DSM-5 (CAPS-5). Nationa Center for PTSD, 2013. http://www.ptsd.va.gov

23. Wittchen HU, Zaudig M, Fydrich T. [Structured Clinical Interview for DSM-IV Axis I Disorders (SCID-I)]. Göttingen: Hogrefe, 1997.

24. Derogatis LR. Brief Symptom Inventory (BSI) 18. Administration, scoring and procedures manual. Minneapolis, MN: NCS Pearson, 2000.

25. Leppert K, Koch B, Brähler E, et al. [Resilience scale-evaluation of a long (RS-25) and a short version (RS-13)]. Klinische Diagnostik und Evaluation 2008:2:226-43.

26. Greenglass ER, Schwarzer R, Taubert, S. The Proactive Coping Inventory (PCl): a multidimensional research instrument. Toronto: York University, 1999. http://estherg.info.yorku.ca/greenglass-pci/

27. Smets EMA, Garssen B, Bonke B, et al. The Multidimensional Fatigue Inventory (MFI): psychometric qualities of an instrument to assess fatigue. J Psychosom Res 1995;39:315-25.

28. Herdman M, Gudex C, Lloyd A, et al. Development and preliminary testing of the new five-level version of EQ-5D (EQ-5D-5L). Qual Life Res 2011;20:1727-36.

29. Deck R, Mittag O, Hüppe A, et al. [Index for Measuring Limitations of Social Participation (IMET) - first results of an ICF-based assessment instrument]. Praxis Klinische Verhaltensmedizin und Rehabilitation 2007;20:113-20.

30. Bodenmann G. [Dyadic Coping Inventory (DCI). Test manual]. Bern: Huber, 2008.

31. Hendrick SS. A generic measure of relationship satisfaction. J Marriage Fam 1988;50:93-8.

32. Wegmann E, Stodt B, Brand M. Addictive use of social networking sites can be explained by the interaction of Internet use expectancies, Internet literacy, and psychopathological symptoms. $J$ Behav Addict 2015;4:155-62.

33. Foa EB, Ehlers A, Clark DM, et al. The Posttraumatic Cognitions Inventory (PTCl): development and validation. Psychol Assess 1999;11:303-14.

34. Mander JV, Wittorf A, Schlarb A, et al. Change mechanisms in psychotherapy: multiperspective assessment and relation to outcome. Psychother Res 2013;23:105-16.

35. Wagnild GM, Young HM. Development and psychometric evaluation of the Resilience Scale. J Nurs Meas 1993;1:165-78.

36. Furukawa TA, Noma $\mathrm{H}$, Caldwell DM, et al. Waiting list may be a nocebo condition in psychotherapy trials: a contribution from network meta-analysis. Acta Psychiatr Scand 2014;130:181-92. 